\title{
Buckling of nonuniform carbon nanotubes under concentrated and distributed axiall loads
}

\author{
Mouafo Teifouet Armand Robinson ${ }^{1,2}$ and Sarp Adali ${ }^{1}$ \\ ${ }^{1}$ Discipline of Mechanical Engineering, University of KwaZulu-Natal, Durban 4041, South Africa \\ ${ }^{2}$ Department of Physics, University of Dschang, Dschang, Cameroon \\ Correspondence to: Sarp Adali (adali@ukzn.ac.za)
}

Received: 6 April 2017 - Revised: 17 July 2017 - Accepted: 25 July 2017 - Published: 9 October 2017

\begin{abstract}
Buckling of nonuniform carbon nanotubes are studied with the axial load taken as a combination of concentrated and axially distributed loads. Constitutive modelling of the nanotubes is implemented via nonlocal continuum mechanics. Problem solutions are obtained by employing a weak formulation of the problem and the Rayleigh-Ritz method which is implemented by using orthogonal Chebyshev polynomials. The accuracy of the method of solution is verified against available results. Solutions are obtained for the cases of uniformly distributed and triangularly distributed axial loads. Contour plots are given to assess the effect of nonuniform crosssections and the small-scale parameter on the buckling load for a combination of simply supported, clamped and free boundary conditions.
\end{abstract}

\section{Introduction}

Superior properties of carbon nanotubes (CNT) led to their use in a number of technologically advanced fields such as biotechnology, nanocomposites and nanoelectronics. Even though CNTs have high stiffness and large failure strain, they are prone to buckling under compressive loads due to their slenderness which results in limiting their use in applications involving compressive axial loads. Thus, in many applications of CNTs, buckling becomes of primary interest as this could be the dominant failure mode. Such applications include nano-mechanical devices, drug delivery and nanocomposites. This resulted in buckling of CNTs being an active area of research for a number of years and the subject has been investigated extensively due to its importance (Elishakoff et al., 2012; Shima, 2012; Wang et al., 2010). Recent works on the buckling of CNTs with uniform cross-sections and subject to a concentrated axial load include Pradhan et al. (2011), Ansari et al. (2011), Hosseini-Ara et al. (2012), Zidour et al. (2014), and Ebrahimi et al. (2016). Studies on the stability of uniform CNTs under distributed axial loads include buckling of CTNs under their own weight (Wang et al., 2004, 2016; Mustapha and Zhong, 2012) and under uniformly and triangularly distributed axial loads (Robinson and Adali, 2016). Nonuniform CNTs are employed in the design of nanostructures such as nanoscale sensors and actuators and their vibration characteristics have been studied in Murmu and Pradhan (2009), Lee and Chang (2010, 2011), and Tang et al. (2014). Studies on the buckling of nonuniform nanotubes seem to have been restricted to nanocones which are of interest in atomic force microscopy and electroanalysis (Chen et al., 2006; Sripirom et al., 2011) as the tip structure of nanocones can be used to achieve mechanical properties which cannot be obtained by uniform nanotubes. A number of studies have been directed to elucidating the mechanical and physical properties of nanocones (Wei et al., 2007; Ansari and Mahmoudinezhad, 2015). Buckling and post-buckling behaviors of nanocones have been studied in Liew et al. (2007), Yan et al. (2013). Molecular mechanics was employed in Fakhrabadi et al. (2012) to investigate the buckling behavior of nanocones and a computational approach was used in Yan et al. (2012) to compute the buckling loads of nanocones. In the above studies buckling loads were specified as concentrated axial loads.

Present study involves the buckling of nonuniform nanotubes under variable axial loads employing a nonlocal continuum model and extends the results of Robinson and Adali (2016) to nonuniform nanotubes. Axial loads acting on the nanotube are taken as a combination of concentrated 
and distributed loads. Distributed loads can be uniform corresponding to self-weight or triangular. The method of solution involves the weak variational formulation of the problem and employing the Rayleigh-Ritz method using orthogonal Chebyshev polynomials. Numerical results are given for various combinations of boundary conditions in the form of contour plots and line graphs to study the effect of the problem parameters on buckling loads.

\section{Nonlocal problem formulation}

In the nonlocal formulation of the constitutive equations of continuum mechanics, the stress at a point depends not only on the strain at that point but also on strains at all other points in the domain. As such stress-strain relations of nonlocal elasticity differs from those of classical elasticity and the general form of these relations are expressed as an integral over the domain (Fernández-Sáez et al., 2016; Taghizadeh et al., 2015). Nonlocal formulation has the advantage of taking into account the small scale effects in the form of a material parameter making it suitable for the study of nano scale components. In the one-dimensional case, differential equation form of the integral constitutive relation can be expressed as

$\sigma(x)-\left(e_{0} a\right)^{2} \frac{\partial^{2} \sigma(x)}{\partial x^{2}}=E \varepsilon(x)$

where $\sigma(x)$ is the stress, $\varepsilon(x)$ the strain, $e_{0} a$ is the small scale parameter and $E$ is the Young's modulus. Bending moment at a point $x$ can be computed as

$M(x)=\iint_{A} z \sigma(x) \mathrm{d} A$

with $A$ denoting the cross-sectional area. Using Eqs. (1) and (2), the differential equation for $M(x)$ can be obtained in terms of the deflection $w(x)$ as

$M-\left(e_{0} a\right)^{2} M^{\prime \prime}=-E I(x) w^{\prime \prime}$

where a prime denotes differentiation with respect to $x$ and $I(x)$ is the moment of inertia of the nonuniform crosssectional area $A(x)$. The classical (local) elasticity equation for $M(x)$ corresponds to Eq. (3) with $e_{0} a=0$. The equation governing the buckling of a nanotube subject to a distributed axial load $N_{i}(x)$ can be expressed in terms of moment $M(x)$ and deflection $w(x)$ as

$M^{\prime \prime}-\left(N_{i}(x) w^{\prime}\right)^{\prime}=0$

In Eq. (2), the compressive load $N_{i}(x)$ acting on the nanotube consists of a concentrated load $P$ and a distributed load $Q_{i}(x)$ as shown in Fig. 1 and can be expressed as

$N_{i}(x)=P+Q_{i}(x), \quad 0 \leq x \leq L$

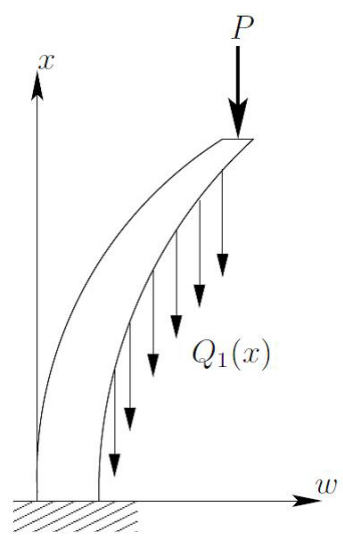

(a)

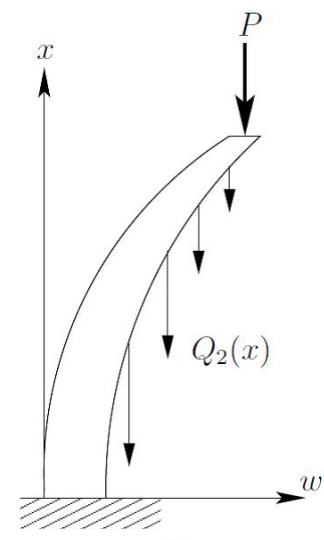

(b)
Figure 1. Clamped-free columns under tip loads and distributed axial loads, (a) uniformly distributed load, (b) triangularly distributed load.

where $L$ is the length of the nanotube. In the present study two different distributed axial loads will be considered, namely, uniformly distributed load $Q_{1}(x)=\bar{q}_{1}(L-x)$ (Fig. 1a) and triangularly distributed load $Q_{2}(x)=\frac{1}{2} \bar{q}_{2}(L-$ $x)^{2}$ (Fig. 1b).

Substituting the second derivative of $M(x)$ from Eq. (4) into Eq. (3), the expression for the nonlocal moment is computed as

$M=-E I(x) w^{\prime \prime}+\left(e_{0} a\right)^{2}\left(N_{i}(x) w^{\prime}\right)^{\prime}$

From Eqs. (4) and (6), the differential equation governing the buckling of a nonuniform nanotube can be obtained as

$$
\begin{aligned}
D(w) & =\left(E I(x) w^{\prime \prime}\right)^{\prime \prime}+\left(N_{i} w^{\prime}\right)^{\prime} \\
& -\left(e_{0} a\right)^{2}\left(N_{i}^{\prime} w^{\prime}+N_{i} w^{\prime \prime}\right)^{\prime \prime}=0
\end{aligned}
$$

\section{Weak formulation}

The weak form of the problem corresponds to an integral expression combining the differential equation and the natural boundary conditions. It provides a suitable approximation technique using polynomials as the approximating functions. Derivation of the weak form of Eq. (7) is outlined next by first noting that

$$
\int_{0}^{L} D(w) w \mathrm{~d} x=0
$$

since $D(w)=0$. Each term in Eq. (8) is expressed as

$$
\sum_{i=1}^{4} U_{i}(w)=0
$$

in order to incorporate the natural boundary conditions into the formulation. In Eq. (9), the expressions for $U_{i}(w)$ are 
Table 1. Comparison of buckling loads $p$ (concentrated load only) with existing results for four boundary conditions with $E I(X)=E I_{0}(1-$ $\beta X)^{4}$ and $\mu=0$ (local beam).

\begin{tabular}{l|ll|ll|ll|l|l}
\hline & \multicolumn{2}{|c|}{ SS } & \multicolumn{2}{c|}{ CS } & \multicolumn{2}{c|}{ CC } & \multicolumn{2}{c}{ CF } \\
\hline$\beta$ & Present & $\begin{array}{c}\text { Wei et } \\
\text { al. (2010) }\end{array}$ & Present & $\begin{array}{c}\text { Wei et } \\
\text { al. (2010) }\end{array}$ & Present & $\begin{array}{l}\text { Wei et } \\
\text { al. (2010) }\end{array}$ & Present & $\begin{array}{c}\text { Wei et } \\
\text { al. (2010) }\end{array}$ \\
\hline 0.0 & 9.869 & 9.870 & 20.191 & 20.191 & 39.478 & 39.478 & 2.467 & 2.467 \\
0.2 & 6.317 & 6.317 & 12.922 & 12.922 & 25.266 & 25.266 & 1.883 & 1.884 \\
0.4 & 3.553 & 3.553 & 7.269 & 7.269 & 14.212 & 14.212 & 1.309 & 1.309 \\
0.6 & 1.579 & 1.579 & 3.231 & 3.230 & 6.317 & 6.316 & 0.7567 & 0.757 \\
0.8 & 0.398 & 0.395 & 0.815 & 0.807 & 1.583 & 1.547 & 0.265 & 0.265 \\
\hline
\end{tabular}

given by

$$
\begin{gathered}
U_{1}(w)=\int_{0}^{L}\left(E I(x) w^{\prime \prime}\right)^{\prime \prime} w \mathrm{~d} x, \\
U_{2}(w)=\int_{0}^{L}\left(N_{i} w^{\prime}\right)^{\prime} w \mathrm{~d} x \\
U_{3}(w)=-\left(e_{0} a\right)^{2} \int_{0}^{L}\left(N_{i}^{\prime} w^{\prime}\right)^{\prime \prime} w \mathrm{~d} x \\
U_{4}(w)=-\left(e_{0} a\right)^{2} \int_{0}^{L}\left(N_{i} w^{\prime \prime}\right)^{\prime \prime} w \mathrm{~d} x
\end{gathered}
$$

Expressions for $U_{i}(w)$ are transformed to integral and boundary terms by integration by parts, viz.,

$$
\begin{aligned}
& U_{1}(w)=\int_{0}^{L} E I(x)\left(w^{\prime \prime}\right)^{2} \mathrm{~d} x \\
& +\left[\left(E I(x) w^{\prime \prime}\right)^{\prime} w-E I(x) w^{\prime} w^{\prime \prime}\right]_{x=0}^{x=L} \\
& U_{2}(w)=-\int_{0}^{L} N_{i}\left(w^{\prime \prime}\right)^{2} \mathrm{~d} x+\left.N_{i} w^{\prime} w\right|_{x=0} ^{x=L} \\
& U_{3}(w)=-\left(e_{0} a\right)^{2} \int_{0}^{L} N_{i}^{\prime} w^{\prime} w^{\prime \prime} \mathrm{d} x \\
& -\left(e_{0} a\right)^{2}\left[\left(N_{i}^{\prime} w^{\prime}\right)^{\prime} w-N_{i}^{\prime} w^{\prime 2}\right]_{x=0}^{x=L} \\
& U_{4}(w)=-\left(e_{0} a\right)^{2} \int_{0}^{L} N_{i} w^{\prime \prime 2} \mathrm{~d} x \\
& -\left(e_{0} a\right)^{2}\left[\left(N_{i} w^{\prime \prime}\right)^{\prime} w-N_{i} w^{\prime \prime} w^{\prime}\right]_{x=0}^{x=L}
\end{aligned}
$$

The moment expression is given by Eq. (6) and the shear force by

$$
\begin{aligned}
& V(x)=\left(E I(x) w^{\prime \prime}\right)^{\prime}+N_{i} w^{\prime} \\
& -\left(e_{0} a\right)^{2}\left[\left(N_{i}^{\prime} w^{\prime}\right)^{\prime}+\left(N_{i} w^{\prime \prime}\right)^{\prime}\right]
\end{aligned}
$$

Then Eq. (8) can be expressed as

$$
\begin{aligned}
& \int_{0}^{L}\left\{E I(x) w^{\prime \prime 2}-N_{i} w^{\prime 2}-\left(e_{0} a\right)^{2}\left[N_{i}^{\prime} w^{\prime} w^{\prime \prime}+N_{i} w^{\prime \prime 2}\right]\right\} \mathrm{d} x \\
& \quad+\left.\left(V(x) w+M(x) w^{\prime}\right)\right|_{x=0} ^{x=L}=0
\end{aligned}
$$

where $M(x)$ and $V(x)$ are defined by Eqs. (4) and (16), respectively. Boundary conditions for various cases can be expressed as follows:

Simply supported boundary conditions:

$$
w(0)=0, M(0)=0, w(L)=0, M(L)=0
$$

Clamped-clamped boundary conditions:

$$
w(0)=0,\left.\frac{\mathrm{d} w}{\mathrm{~d} x}\right|_{x=0}=0, w(L)=0,\left.\frac{\mathrm{d} w}{\mathrm{~d} x}\right|_{x=L}=0
$$

Clamped-simply supported boundary conditions :

$$
w(0)=0,\left.\frac{\mathrm{d} w}{\mathrm{~d} x}\right|_{x=0}=0, w(L)=0, M(L)=0
$$

Clamped-free supported boundary conditions :

$$
w(0)=0,\left.\frac{\mathrm{d} w}{\mathrm{~d} x}\right|_{x=0}=0, M(L)=0, V(L)=0
$$

Let $I(x)=I_{0} g(x)$ where $I_{0}$ is a dimensional reference constant and $g(x)$ is a nondimensional function of $x$. Nondimensional form of the formulation can be obtained by introducing the dimensionless variables defined as

$$
\begin{gathered}
X=\frac{x}{L} \quad W=\frac{w}{L} \quad \mu=\frac{e_{0} a}{L} \quad p=\frac{P L^{2}}{E I_{0}} \\
q_{i}=\frac{\bar{q}_{i} L^{2+i}}{E I_{0}} \quad n_{i}=\frac{N_{i} L^{2}}{E I_{0}}
\end{gathered}
$$


Table 2. Comparison of buckling loads under distributed loads with existing results for four boundary conditions with $\mu=0$ (local beam).

\begin{tabular}{llll|ll}
\hline & \multicolumn{3}{c|}{$q_{1}$} & & $q_{2} / 2$ \\
\hline BC & Present & $\begin{array}{l}\text { Duan and } \\
\text { Wang (2008) }\end{array}$ & $\begin{array}{l}\text { Wang et } \\
\text { al. (1988) }\end{array}$ & Present & $\begin{array}{l}\text { Eisenberger } \\
(1991)\end{array}$ \\
\hline SS & 18.569 & 18.569 & 18.58 & 23.239 & 23.239 \\
CS & 52.504 & 52.501 & 53.91 & 78.983 & 78.983 \\
CC & 74.643 & 74.629 & 78.96 & 107.823 & 107.823 \\
CF & 7.837 & 7.837 & 7.84 & 16.101 & 16.101 \\
\hline
\end{tabular}

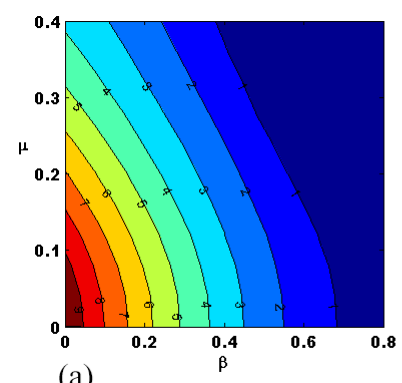

(a)

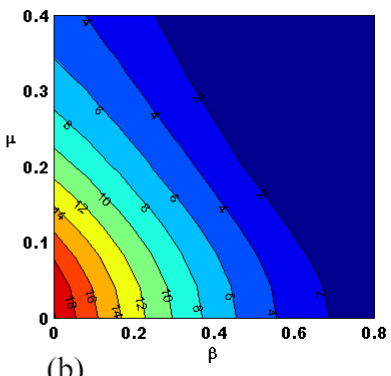

(b)

Figure 2. Contour plot of $p$ with respect to $\beta$ and $\mu$, (a) SS, (b) CS.

Nondimensional form of Eq. (16) can be expressed as

$$
\begin{aligned}
& \int_{0}^{1}\left\{g(X) W^{\prime \prime 2}-\eta_{i} W^{\prime 2}-\mu^{2}\left[\eta_{i}^{\prime} W^{\prime} W^{\prime \prime}+\eta_{i} W^{\prime \prime 2}\right]\right\} \mathrm{d} x \\
& \quad+\left.\left(v(X) W+m(X) W^{\prime}\right)\right|_{X=0} ^{X=1}=0
\end{aligned}
$$

where

$$
\begin{aligned}
& m(X)=\frac{L}{E I_{0}} M=-g(X) W^{\prime \prime}+\mu^{2}\left(n_{i} W^{\prime}\right)^{\prime} \\
& v(X)=\frac{L^{2}}{E I_{0}} V(x)=\left(g(X) W^{\prime \prime}\right)^{\prime}+\eta_{i} W^{\prime} \\
& \quad-\mu^{2}\left[\left(\eta_{i}^{\prime} W^{\prime}\right)^{\prime}+\left(\eta_{i}^{\prime} W^{\prime \prime}\right)^{\prime}\right] \\
& n_{1}(X)=p+q_{1}(1-X), \quad n_{2}(X)=p+\frac{1}{2} q_{2}(1-X)^{2}
\end{aligned}
$$

\section{Method of solution}

Polynomial approximation of the solution is obtained by implementing the Rayleigh-Ritz method which involves approximating the non-dimensional deflection function $W(X)$ in terms of Chebyshev polynomials. Accurate results can be obtained as the approximating polynomials are complete in the function space and convergence is tested. To satisfy the geometric boundary conditions, Chebyshev polynomials are multiplied by suitable boundary functions corresponding to the specific boundary condition. Deflection $W(X)$ is ex-
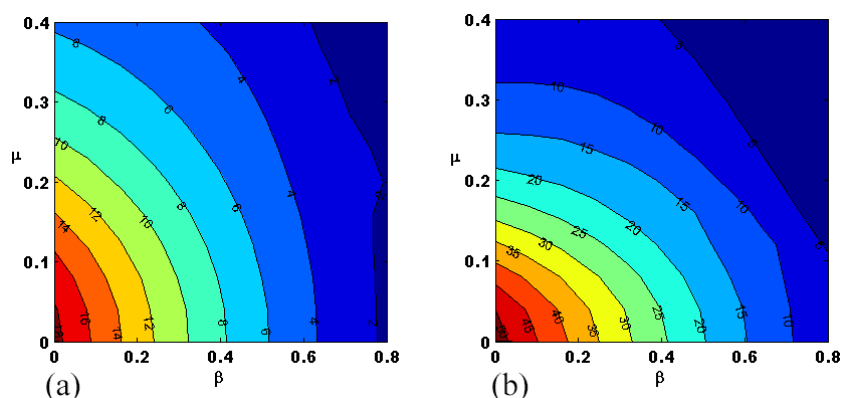

(b)

Figure 3. Contour plot of $q_{1}$ with respect to $\beta$ and $\mu$, (a) SS, (b) CS.

pressed as

$W(X)=X^{r}(1-X)^{s} \sum_{j=1}^{N} c_{j} f_{j-1}(X)$

where $r$ and $s$ take the values 0,1 or 2 for free, simply supported and clamped boundaries, respectively. Parameters $c_{j}$ are determined as part of the solution of an eigenvalue problem which yields the buckling load as the minimum eigenvalue. In Eq. (22), $f_{j}(X)$ is the $j$ th Chebyshev polynomial with $f_{0}(X)=1$ and $f_{1}(X)=X$. The remaining terms are obtained from

$f_{j+1}(X)=2 X f_{j}(X)-f_{j-1}(X)$

To verify the accuracy of the present method, it was applied to the buckling of a nonuniform column subject to a tip load only, i.e., $p>0$ and $q(x)=0$, as given in Duan and Wang (2008). The column has a square cross-section and its stiffness is given by $E I(X)=E I_{0}(1-\beta X)^{4}$ (Wei et al., 2010). The results are given in Table 1 . It is observed that the present method implemented by using Chebyshev polynomials give accurate results. Next the method is applied to columns subject to distributed axial loads and the results are shown in Table 2. The present method is observed to be accurate also in the case of buckling with distributed axial loads.

\section{Numerical results}

Numerical results are given for the boundary conditions SS, $\mathrm{CS}, \mathrm{CC}$ and $\mathrm{CF}$ which are given by Eqs. (17)-(20). The range 


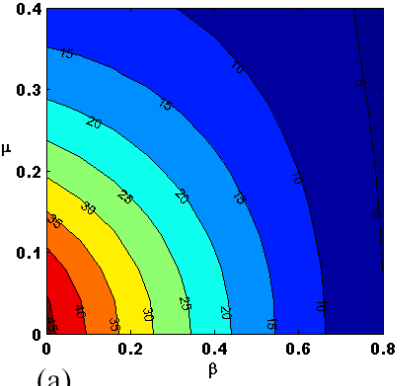

(a)

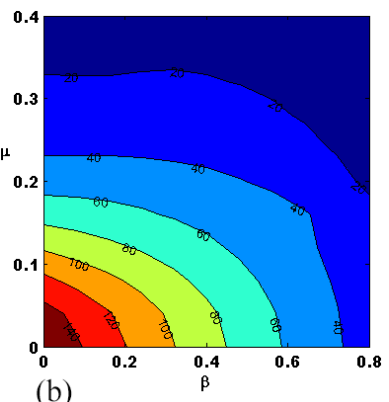

(b)

Figure 4. Contour plot of $q_{2}$ with respect to $\beta$ and $\mu$, (a) SS, (b) CS.

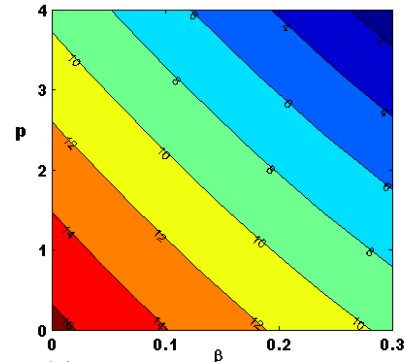

(a)

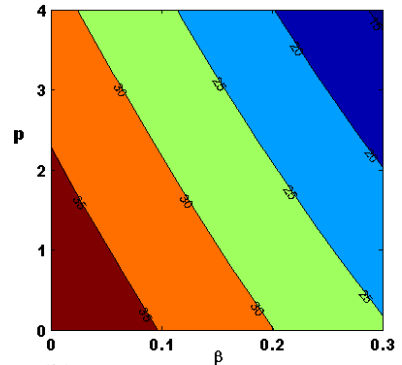

(b)

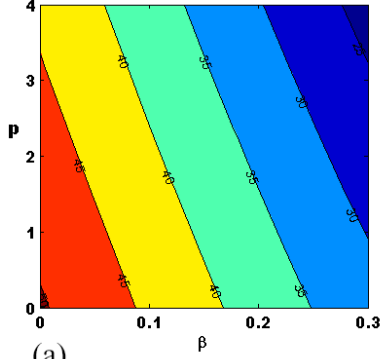

(a)

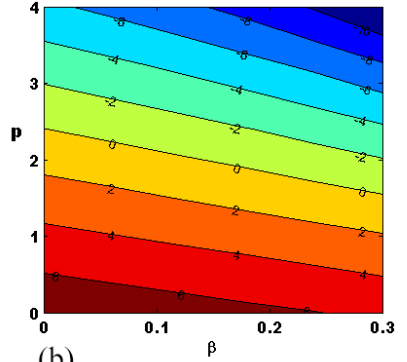

(b)
Figure 6. Contour plot of $q_{1}$ with respect to $p$ and $\beta$ with $\mu=0.1$, (a) CC, (b) $\mathrm{CF}$.

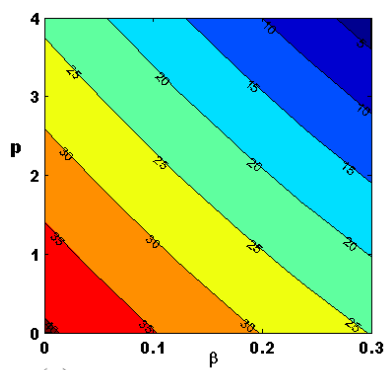

(a)

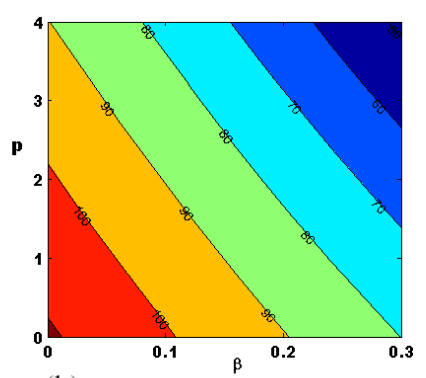

(b)
Figure 5. Contour plot of $q_{1}$ with respect to $p$ and $\beta$ with $\mu=0.1$, (a) SS, (b) CS.

of the small scale parameter $\mu$ is taken as $0 \leq \mu \leq 0.4$. The cross-section is specified as a square and the moment of inertia is taken as $I(X)=I_{0}(1-\beta X)^{4}$. The contour plots of the buckling load $p$ with respect to $\mu$ and $\beta$ are shown in Fig. 2 for simply supported and clamped-hinged nanocolumns. It is observed that the buckling load decreases as the small-scale parameter increases. The corresponding results for uniformly distributed axial load and triangularly distributed axial load are shown in Figs. 3 and 4, respectively. It is observed that, the effect of the non-uniformity parameter $\beta$ on the buckling load is more pronounced for the concentrated load $p$.

Next the buckling under the combined axial loads of a concentrated load $p$ and a distributed load is investigated. Contour plots for the buckling load $q_{1}$ corresponding to the uniformly distributed axial load are given in Fig. 5 with respect to $p$ and $\beta$ for simply supported and clampedhinged nanocolumns and in Fig. 6 for clamped-clamped and clamped-free nanocolumns with $\mu=0.1$.

Corresponding results for $q_{2}$ (triangularly distributed axial load) are given in Figs. 7 and 8. Figures 5-8 show the numerical differences in the buckling loads in the case of uniformly and triangularly distributed axial loads for nonuniform nanocolumns. The effect of the boundary conditions on the buckling loads can be observed from these figures. Buckling parameters $q_{1}$ and $q_{2}$ are least affected by the change in the stiffness $E I(x)$ as indicated by $\beta$ in the case of clampedfree columns (Figs. $6 \mathrm{~b}$ and $8 \mathrm{~b}$ ) and most affected in the case of clamped-clamped columns (Figs. 6a and 8a). Similarly,
Figure 7. Contour plot of $q_{2}$ with respect to $\beta$ and $p$ with $\mu=0.1$, (a) SS, (b) CS.

the buckling loads $q_{1}$ and $q_{2}$ decrease most by an increase in the tip load $p$ in the case of clamped-free columns as expected (Figs. 6b and $8 \mathrm{~b}$ ). In fact $q_{1}$ and $q_{2}$ become negative, i.e., change from compression to tension, above a certain value of $p$ (Figs. $6 \mathrm{~b}$ and $8 \mathrm{~b}$ ).

\section{Conclusions}

Buckling of nonuniform nanotubes subject to concentrated and variable axial loads was studied. In particular, uniformly distributed and triangularly distributed axial loads and nonuniform shapes with moment of inertia proportional to $(1-\beta X)^{4}$ were investigated. The results are obtained by Rayleigh-Ritz method employing Chebyshev polynomials of first kind as the approximating functions for a combination of simply supported, clamped and free boundary conditions. The accuracy of the method was verified by comparing the solutions with available results in the literature. Chebyshev polynomials are used extensively in the solution of engineering problems due to their fast convergence and accuracy as compared to other orthogonal functions as noted in Sari and Butcher (2010), Filippi et al. (2015). Moreover they are easy to programme in symbolic form and the required accuracy can be attained by the number of polynomials (Sari and Butcher, 2010).

The effects of non-uniformity of the cross-section and the small-scale parameter on the buckling loads were investi- 


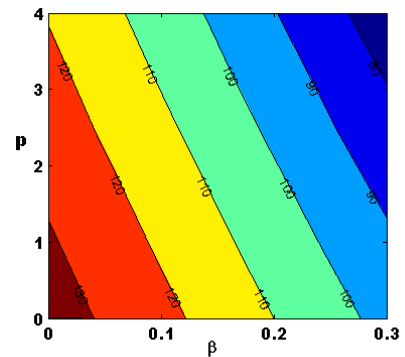

(a)

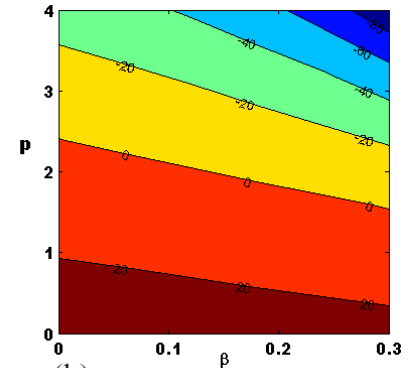

(b)
Figure 8. Contour plot of $q_{2}$ with respect to $\beta$ and $p$ with $\mu=0.1$, (a) $\mathrm{CC}$, (b) $\mathrm{CF}$.

gated by means of contour plots. These plots indicate the sensitivity of the buckling loads to problem parameters and it was observed that buckling load under concentrated tip load is more sensitive to the change in the cross-section. On the other hand buckling load is more sensitive to the magnitude of the tip load for the clamped-free boundary conditions.

Data availability. All the data for this paper are given in the form of tables and figures.

Competing interests. The authors declare that they have no conflict of interest.

Acknowledgements. The research reported in this paper was supported by research grants from the University of KwaZulu-Natal (UKZN) and from National Research Foundation (NRF) of South Africa. The authors gratefully acknowledge the supports provided by UKZN and NRF.

Edited by: Anders Eriksson

Reviewed by: two anonymous referees

\section{References}

Ansari, R. and Mahmoudinezhad, E.: Characterizing the mechanical properties of carbon nanocones using an accurate spring-mass model, Comput. Mat. Sci., 101, 260-266, doi:10.1016/j.commatsci.2015.02.001, 2015.

Ansari, R., Sahmani, S., and Rouhi, H.: Rayleigh-Ritz axial buckling analysis of single-walled carbon nanotubes with different boundary conditions, Phys. Lett. A, 375, 1255-1263, doi:10.1016/j.physleta.2011.01.046, 2011.

Chen, C., Chen, L. H., Ye, X. R., Daraio, C., Jin, S., Orme, C. A., Quist, A., and Lal, R.: Extreme sharp carbon nanocone probes for atomic force microscopy imaging, Appl. Phys. Lett., 88, 153102, doi:10.1063/1.2193435, 2006.

Duan, W. H. and Wang, M.: Exact solution for buckling of columns including self-weight, ASCE J. Eng. Mech., 134, 116-119, doi:10.1061/(ASCE)0733-9399(2008)134:1(116), 2008.
Ebrahimi, F., Shaghaghi, G. R., and Boreiry, M.: A semianalytical evaluation of surface and nonlocal effects on buckling and vibrational characteristics of nanotubes with various boundary conditions, Int. J. Struct. Stab. Dy., 16, 1550023, doi:10.1142/S0219455415500236, 2016.

Eisenberger, M.: Buckling loads for variable cross-section members with variable axial forces, Int. J. Solids Struct., 27, 135-144, doi:10.1016/0020-7683(91)90224-4, 1991.

Elishakoff, I., Pentaras, D., Dujat, K., Versaci, C., Muscolino, G., Storch, J., Bucas, S., Challamel, N., Natsuki, T., Zhang, Y. Y., Wang, C. M., and Ghyselinck, G.: Carbon Nanotubes and Nanosensors: Vibrations, Buckling and Ballistic Impact, ISTE London and John Wiley, New Jersey, 2012.

Fakhrabadi, M. M. S., Khani, N., Omidvar, R., and Rastgoo, A.: Investigation of elastic and buckling properties of carbon nanocones using molecular mechanics approach, Comput. Mat Sci., 61, 248-256, doi:10.1016/j.commatsci.2012.04.029, 2012.

Fernández-Sáez, J., Zaera, R., Loya, J.A., and Reddy, J.N.: Bending of Euler-Bernoulli beams using Eringen's integral formulation: A paradox resolved, Int. J. Eng. Sci., 99, 107-116, doi:10.1016/j.ijengsci.2015.10.013, 2016.

Filippi, M., Pagani, A., Petrolo, M., Colonna, G., and Carrera, E.: Static and free vibration analysis of laminated beams by refined theory based on Chebyshev polynomials, Compos. Struct., 132, 1248-1259, doi:10.1016/j.compstruct.2015.07.014, 2015.

Hosseini-Ara, R., Mirdamadi, H. R., and Khademyzadeh, H.: Buckling analysis of short carbon nanotubes based on a novel Timoshenko beam model, J. Theor. Appl. Mech., 50, 975986, available at: http://www.ptmts.org.pl/jtam/index.php/jtam/ article/view/v50n4p975/157, 2012.

Lee, H. L. and Chang, W. J.: Surface and small-scale effects on vibration analysis of a nonuniform nanocantilever beam, Physica E, 43, 466-469, doi:10.1016/j.physe.2010.08.030, 2010.

Liew, K. M., Wei, J. X., and He, X. Q.: Carbon nanocones under compression: Buckling and post-buckling behaviors, Phys. Rev. B, 75, 195435, doi:10.1103/PhysRevB.75.195435, 2007.

Murmu, T. and Pradhan, S. C.: Small-scale effect on the vibration of nonuniform nano cantilever based on nonlocal elasticity theory, Physica E, 41, 1451-1456, doi:10.1016/j.physe.2009.04.015, 2009.

Mustapha, K. B. and Zhong, Z. W.: Stability of single-walled carbon nanotubes and single-walled carbon nanocones under selfweight and an axial tip force, Int. J. Eng. Sci., 50, 268-278, doi:10.1016/j.ijengsci.2010.12.006, 2012.

Pradhan, S. and Reddy, G. K.: Buckling analysis of single walled carbon nanotube on Winkler foundation using nonlocal elasticity theory and DTM, Comput. Mater. Sci., 50, 1052-1056, doi:10.1016/j.commatsci.2010.11.001, 2011.

Robinson, M. T. A. and Adali, S.: Variational solution for buckling of nonlocal carbon nanotubes under uniformly and triangularly distributed axial loads, Compos. Struct., 156, 101-107, doi:10.1016/j.compstruct.2016.01.026156, 2016.

Sari, M. and Butcher, E. A.: Natural frequencies and critical loads of beams and columns with damaged boundaries using Chebyshev polynomials, Int. J. Eng. Sci., 48, 862-873, doi:10.1016/j.ijengsci.2010.05.008, 2010.

Shima, H.: Buckling of carbon nanotubes: A state of the art review, Materials, 5, 47-84, doi:10.3390/ma5010047, 2012. 
Sripirom, J., Noor, S., Köhler, U., and Schulte, A.: Easily made and handled carbon nanocones for scanning tunneling microscopy and electroanalysis, Carbon, 49, 2402-2412, doi:10.1016/j.carbon.2011.02.007, 2011.

Taghizadeh, M., Ovesy, H. R., and Ghannadpour, S. A. M.: Nonlocal integral elasticity analysis of beam bending by using finite element method, Struct. Eng. Mech., 54, 755-769, doi:10.12989/sem.2015.54.4.755, 2015.

Tang, H. L., Shen, Z. B., and Li, D. K.: Vibration of nonuniform carbon nanotube with attached mass via nonlocal Timoshenko beam theory, J. Mech. Sci. Technol., 28, 3741-3747, doi:10.1007/s12206-014-0835-6, 2014.

Wang, C. M. and Ang, K. K.: Buckling capacities of braced heavy columns under axial loads, Comput. Struct., 28, 563-571, doi:10.1016/0045-7949(88)90002-8, 1988.

Wang, C. M., Zhang, H., Challamel, N., and Xiang, Y.: Buckling of nonlocal columns with allowance for selfweight, ASCE J. Eng. Mech., 142, 04016037, doi:10.1061/(ASCE)EM.19437889.0001088, 2016.

Wang, C. M., Zhang, Y. Y., Xiang, Y., and Reddy, J. N.: Recent studies on buckling of carbon nanotubes, Appl. Mech. Rev., 63, 030804-030818, doi:10.1115/1.4001936, 2010.

Wang, G. W., Zhao, Y. P., and Yang, G. T.: The stability of a vertical single-walled carbon nanotube under its own weight, Mater. Design, 25, 453-457, doi:10.1016/j.matdes.2004.01.003, 2004.
Wei, D. J., Yan, S. X., Zhang, Z. P., and Li, X.-F.: Critical load for buckling of non-prismatic columns under selfweight and tip force, Mech. Res. Commun., 37, 554-558, doi:10.1016/j.mechrescom.2010.07.024, 2010.

Wei, J. X., Liew, K. M., and He, X. Q.: Mechanical properties of carbon nanocones, Appl. Phys. Lett., 91, 261906, doi:10.1063/1.2813017, 2007.

Yan, J. W., Liew, K. M., and He, L. H.: A mesh-free computational framework for predicting buckling behaviors of single-walled carbon nanocones under axial compression based on the moving Kriging interpolation, Comput. Method. Appl. M., 247-248, 103-112, doi:10.1016/j.cma.2012.08.010, 2012.

Yan, J. W., Liew, K. M., and He, L. H.: Buckling and post-buckling of single-wall carbon nanocones upon bending, Compos. Struct., 106, 793-798, doi:10.1016/j.compstruct.2013.07.007, 2013.

Zidour, M., Daouadji, T. H., Benrahou, K. H., Tounsi, A., Adda Bedia, El A., and Hadji, L.: Buckling analysis of chiral single-walled carbon nanotubes by using the nonlocal Timoshenko beam theory, Mech. Compos. Mat., 50, 95-104, doi:10.1007/s11029-014-9396-0, 2014. 